\section{RELATION BETWEEN CHILD ABUSE BY PARENTS IN CHILDREN WITH CHRONIC ILLNESS AND SOME OF THE DEMOGRAPHICS CHARACTERS}

\author{
M. Golchin, S. Ziaei, H. Naji, N. Safari
}

School of Nursing and Midwifery - Pediatrics Group, Isfahan University of Medical Sciences, Esfahah, Iran

Background and aims: Child abuse is one of the most common health and social problem. The aim of this study was to determine relation between child abuse by parents with chronic illness and some of the demographics characters.

Methods: This is a descriptive-comparative study. One hundred and six 11-18 years old children with a chronic illness(such as Asthma, epilepsy, leukemia, diabetes and etc)referred to health care center of Isfahan university of medical science were selected by convenient sampling. A questionnaire including demographic data and child abuse scale consist of questions in 4 domains (neglect, emotional, physical and sexual) used for data collection. The questionnair validated by content validity and reliability of questionnaires was estimated 0.78 by Cronbach's alpha.Data analysis were done by SPSS and using t test,correlation coefficient Pierson and spearman.

Results: Result contained a relation between sex, physical and emotional abuse $(p<0.05)$. A direct relation between age and neglect $(p=0.002)$ and also a direct relation between duration of disease and sexual abuse $(p=0.01)$, but there was a reverse relation between number of days of being Absent from school and neglect,physical abuse and total score of abuse $(p<0.05)$. Also result showed that there was a significant relation between neglect and total score of being abused and father's addiction.

Conclusion: Significant meaningful between demographic characters such as, sex, age and duration of children's illness with child abuse in one side and parent's drug addiction on the other side was found.This reveals that some cultural points in community gives an important role to chronic illness in children to prepare doing abuse.

\section{PARENT STRESS AND COPING DURING CHILD HOSPITALISATION: THE STAYCLOSE STUDY}

\author{
J. Wray ${ }^{1,2}$, K. Lee ${ }^{1}$, N. Dearmun ${ }^{3}$, L. Franck ${ }^{1,2}$ \\ ${ }^{1}$ UCL Institute of Child Health, ${ }^{2}$ Great Ormond \\ Street Hospital for Children NHS Trust, London, \\ ${ }^{3}$ Oxford Children's Hospital, Oxford, UK
}

Background and aims: Parental participation in children's hospital care is now considered essential for children's psychosocial wellbeing, yet there is surprisingly little research on parental stress and coping. We are conducting a comprehensive evaluation of the parental experience and the perceived impact of hospital facilities/services on parental adjustment. This abstract reports preliminary findings on parent stress and coping and potential influencing factors.

Methods: Parents/carers of children in a tertiary children's hospital were invited to complete validated measures of mood, coping, stress and social support after admission, discharge and 3 months after discharge.

Results: Baseline questionnaires were completed by parents/carers of 28 hospitalised children, aged 0.1-14.0 years,18 of whom completed questionnaires at discharge. Almost two-thirds of parents scored in the borderline/clinical range for anxiety at baseline and discharge. Higher anxiety scores were associated with the use of denial $(r=.414 ; p=.025)$ and self blame $(r=.519 ; p=.004)$ and a greater number of previous hospital stays $(r=.406$; $p=.085$ ). The behaviour and appearance of their children was the source of greatest hospital-related stress for parents. Three months after discharge $42 \%$ had borderline/clinical levels of anxiety, with baseline anxiety scores significantly correlated with those at discharge and 3 months post-discharge $(r>.7, p<.005)$.

Conclusions: These findings suggest that parents experience substantial stress when their child is hospitalised. Further research is needed to confirm these findings and explore the influence of hospital services and facilities on parental coping. Screening for those at high risk for anxiety and implementing interventions to reduce maladaptive coping strategies may be beneficial. 